\title{
Experimental demonstration of singular-optical colouring of regularly scattered white light
}

\section{O. V. Angelsky \\ oleg@optical.chernovtsy.ua}

\section{S. G. Hanson}

\section{P. P. Maksimyak}

\section{A. P. Maksimyak}

\section{A. L. Negrych}

Department of Correlation Optics, Chernivtsi National University, Chernivtsi 58012, Ukraine

DTU Fotonik, Department of Photonics Engineering, DK-4000 Roskilde, Denmark

Department of Correlation Optics, Chernivtsi National University, Chernivtsi 58012, Ukraine

Department of Correlation Optics, Chernivtsi National University, Chernivtsi 58012, Ukraine

Experimental interference modelling of the effects of colouring of a beam traversing a light-scattering medium is presented. It is shown that the result of colouring of the beam at the output of the medium depends on the magnitudes of the phase delays of the singly forward scattered partial signals. The colouring mechanism has for the first time experimentally been illustrated for a forward propagating beam through a light-scattering medium. This is showed in video-fragments of the interferograms recorded within the zero interference fringe with a gradual change of the path difference of the interfering polychromatic wave trains. Spectral investigation of the effects of colouring has been carried out using a solution of liquid crystal in a polymer matrix. The amplitude ratio of the non-scattered and the singly forward scattered interfering components significantly affects the colour intensity. It has further been established that the spectral content of the illuminating beam strongly influences the colour of the resulting radiation. [DOI: 10.2971/jeos.2008.08029]

Keywords: coherence, polarimetry, statistical optics, optical diagnostics for medicine

\section{INTRODUCTION}

Application of ideas and approaches from coherent singular optics for study of polychromatic incoherent fields allows us to predict, diagnose and investigate the amplitude singularities of the spectral components of such a field and the associated spectral anomalies caused by these singularities [1]-[5]. New approaches have been proposed and implemented for the diagnostics of spatially distributed singularities in polychromatic speckle fields after passing crystals etc. [6]-[9]. In parallel, with some delay, spectral anomalies resulting from coaxial interference in white light due to temporal correlation has become the subject of intense studies $[4,10,11]$.

Revived interest in the study of spectral anomalies under coaxial interference in white light due to temporal correlation is caused by introduction of concepts from coherent singular optics in the area of incoherent polychromatic fields, as well as by the new feasibilities for application of incoherent white light for creation of micromanipulators, optical tweezers etc. To the best of our knowledge, the interference colouring of the sun and the moon observed through a light-scattering medium and described in the 19th and the 20th centuries $[12,13]$ are the forerunners in the historical chain of anomalous spectral phenomena. Later, the same effects of colouring of the image of a lamp filament observed through a grinded plate have been observed and described in [14]. In recent papers devoted to this topic $[10,11]$, variations in explaining the interference, the singular-optical origin of this phenomenon have been proposed. In a recent paper [4] the spectral anomalies observed with a setup based on interference with temporal correlation in white light have been described.

Nevertheless, there is until now no unified approach to the description of these phenomena, though their nature solely relies on optical interference. An experimental model of the mentioned colouring phenomena including a study of possible manifestations would be a constructive step towards solving this problem.

\section{THE APPROACH}

It is known that the mechanism of colouring of a beam passing a light-scattering medium, irrespectively of the nature of this medium, can be divided into several steps:

1. division of the illuminating beam into two components, i.e. an unperturbed field and a singly forward scattered field with some phase delay;

2. interference of non-scattered with the forward scattered part of the radiation, when the zero interference fringe is observed in the resulting field; 
3. colouring of the resulting radiation due to subtracting from the spectrum of the illuminating beam the spectral component for which the average phase difference of the interfering beams is close to $\pi+2 n \pi$.

The phase delays of the scattered partial beams are due to the nature and structure of the light-scattering medium and can be caused either by re-radiation from small light-scattering particles or by difference of the refractive index of larger particles from the refractive index of the surrounding medium $[12,13]$. In case of a grinded glass, the reason for phase delays are mechanisms similar to the ones observed in anti-reflecting optical coatings [11]. One can assume that the colouring of the resulting beam will depend on the spectral content of the illuminating beam, on the phase delay in the forward scattered radiation, as well as on the amplitude ratio of the regular and forward-scattered beams. Unsurprisingly, the intensity of the background caused by the part of radiation that has experienced multiple scattering and is detected by the receiver within the analyzed beam strongly influences the colouring efficiency, i.e. the spectral modulation depth.

Within the framework of the considered approach, the simple way for study of spectral colouring of the forward scattered radiation consists in interfering coaxially propagated waves in a Michelson interferometer following the technique described in a previous article [15]. It is important that the chosen experimental arrangement enables us to model situations with gradually changed phase delays for various amplitude ratios and spectral contents of the radiation.

\section{EXPERIMENT}

The study is carried out using the Michelson's interferometer shown in Figure 1. An incoherent source provides the radiation, $\mathrm{S}$. To form the desired wavelength spectrum, we use a set of certified filters. In the interferometer, a polychromatic field formed by a micro-objective is mixed with a polychromatic reference field. Radiation from the source $S$ is projected onto the $20 \mu \mathrm{m}$ - diameter diaphragm, D, positioned at the focus of the objective $\mathrm{O} 3$ (focal length $200 \mathrm{~mm}$ ) by the objectives $\mathrm{O} 1$ and $\mathrm{O} 2$. In this way, we form a white-light beam with a degree of spatial (transverse) coherence of $95 \%$. The beam-splitter BS divides the incident beam into a reference and an object beam. The mirror M2 with the micro-objective MO2 is shifted along the direction of propagation of the beam, facilitating a controlled change in the optical path delay in the interferometer. An optical phase difference (within a wavelength) is provided by a piezo-ceramic translator, PC, mounted with a mirror M2. By moving one of the mirrors along the direction of propagation of the beam, one can detect the resulting field and record video sequences using a CCD-camera.

The experimental results obtained with coaxial interference of the two fields of equal amplitudes, when a lamp $S$, whose spectrum is shown in Figure 2(a), is used as the radiation source. The results are presented as the fragment of the film (Figure 2(c)) illustrating the effects of colouring by changing the phase difference of the interfering beams within the coherence length of the radiation. To have a notion on the mag-

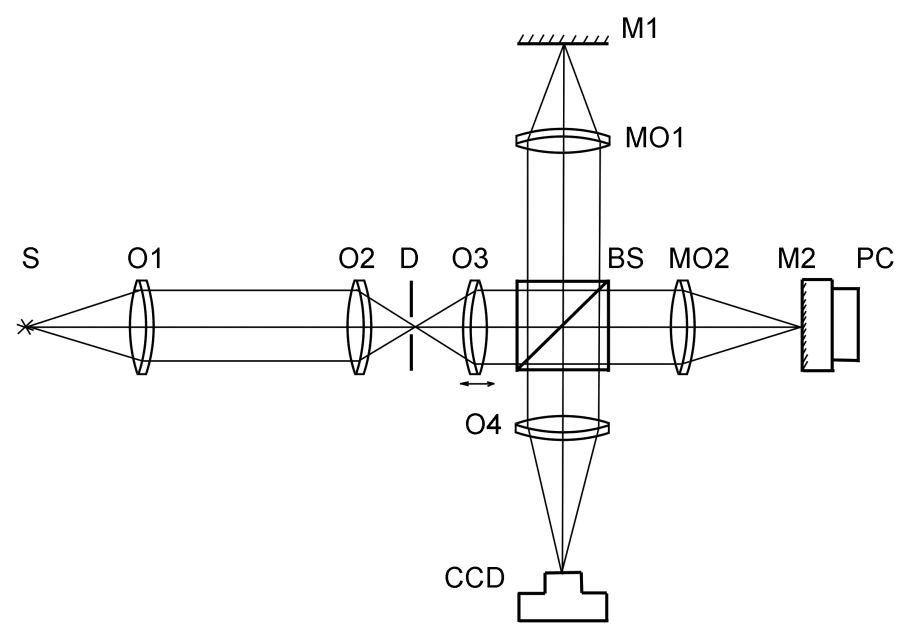

FIG. 1 Experimental arrangement: $\mathrm{S}$-source; $01,02, \mathrm{O}_{3}$ and $\mathrm{O}_{4}$ - objectives; $\mathrm{M} 1, \mathrm{M}_{2}$ - mirrors; D - diaphragm; BS - beam-splitter; $\mathrm{MO} 1$ and M02 - micro-objectives; PC piezo-ceramics.

nitude of path difference between the waves corresponding to some colour in the resulting pattern at the zero interference fringe, one can consider interferograms obtained with the same beams but converging at an angle different from zero (Figure 2(b)). The extent of an interference pattern here corresponds to the path difference that is equal to the coherence length (length of wave train). A priory estimating the coherence length, one can also find the path difference between the waves corresponding to some colour in the case of co-axial interference. In other words, the obtained video-fragments can be digitized both in temporal scale and in magnitudes of path difference of interfering waves. This facilitates a visual estimation of the colour range of the fringes, based on the spectrum of the used radiation. As a matter of fact, interference of two plane waves of equal amplitudes converging at a small angle provides a gradual change of the phase difference between the components at the observation plane. This results in a spatial separation of the amplitude zeroes in the interference distributions of the corresponding spectral components. Actually, different harmonic interference distributions of the resulting amplitude when passing through amplitude zeroes and by changing the phase by $\pi$ gives rise to different spectral components. Thus, each of these distributions is shifted with respect to the distributions for the other components. As a result, the chain of spatially separated vortices (phase singularities) of the spectral components is obtained in analogy with the results obtained for polychromatic speckle fields at the focusing zones of such fields [1, 2,7]. One can investigate the amplitude zeroes in the obtained distributions using one of the techniques described in [6]-[8] or visually, as it is made in [2] by estimation of the colour gamut (spectral content) of the used radiation. These feasibilities appear due to the spatial separation for both the amplitude zeroes and maxima of the spectral components. One observes the presence of pronounced "red" and "blue" shifts in the resulting radiation, which is in accordance with previous results $[16,17]$ and in accordance with a recent experimental study [4]. At the same time, one notices the presence of intense green colouring due to the anomalies in the spectrum of the resulting beam, though the cause for the presence of green is quite obvious. As a matter of fact, for some relative longitudinal shift of the path dif- 
(a)
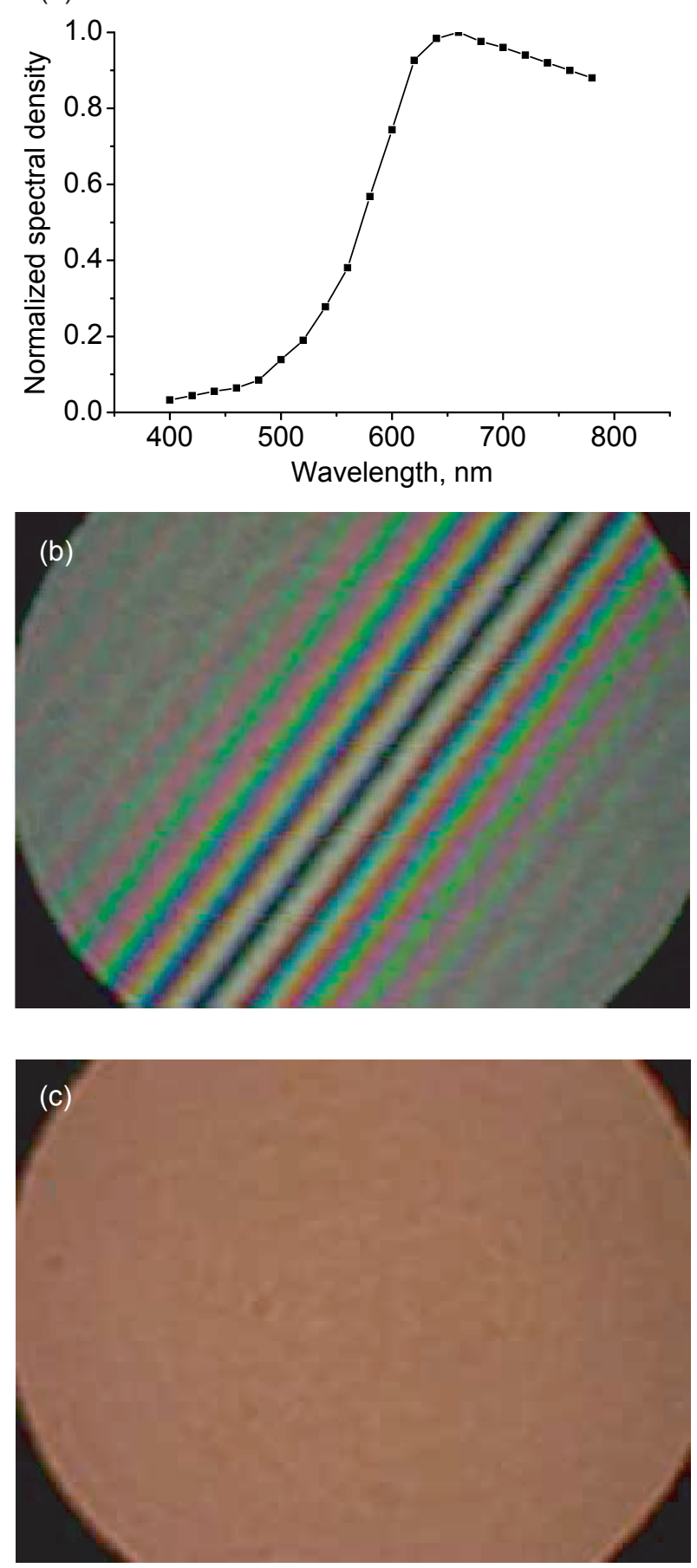

FIG. 2 (a) Spectral content of the used radiation sources. (b) Interferograms obtained with parallel beams in the situation when the angle of convergence of the two waves is different from zero. The presented distributions of fringes qualitatively characterize the spectral content of the interfering beams. The set of colours in the fringes corresponds to the set of colours in a video (Figure $2(\mathrm{c})$ ) and to the spectrum of the used radiation. (c) Video showing the interference pattern when the optical path difference is comparable with the length of the wave train. The limits of the relative longitudinal scanning of the wave trains are comparable with their lengths. (videosize: $3.9 \mathrm{MB}$, format: mov, see Fig2c.mov).

ference, violet and red components may be extinguished by simultaneous destructive interference. This is so, while the spectral component of the red domain undergoes a phase difference of $\pi$ (for an optical path difference of $\lambda / 2$ ), and the spectral component of the violet domain undergoes a phase difference of $3 \pi$ (for an optical path difference of $3 \lambda / 2$ ). As a matter of fact, the intensity distribution of the spectral component of the used source is not uniform. The intensity maxima are in the central and far parts of the spectrum. That is why, green colouring is pronounced when violet and red spectral components are simultaneously extinguished due to interference. Generally speaking, the intensity of colouring is determined by the amplitude ratio of the interfering components and by the intensity of the background.

The presented results support the fact that for non-equality of the amplitudes of the interfering spectral components, the intensity of colouring of the resulting radiation decreases considerably. This is seen from analyzing the colours of the resulting beam for large relative shifts comparable with the length of the interfering wave trains. The same results have been obtained for small relative longitudinal shifts of the wave trains, when their amplitudes were essentially different. These facts explain why previous results in the study of the colouring of radiation transmitted through grinded glasses with different degree of roughness were not reported early [10]. Green-toturquoise colouring of the resulting beam could only be diagnosed visually. In principle, the limits of colouring of the resulting beam are determined by the ratio of the coherence length of radiation and the path difference of the interfering beams. Increasing of path difference of the interfering components will decrease the half-width of the spectrum of the components, which are interferentionally subtracted from the spectrum of the illuminating beam. Complete disappearance of the colouring effect appears when the relative delay exceeds the coherence length. Even in this case, interference subtraction from the spectrum of the illuminating beam of narrow (in spectral sense) quasi-monochromatic components can take place, but observation of this effect is impossible. As a matter of fact, the train is formed by a set of monochromatic components infinitely extended in time. It is natural to assume that such components can interfere even for large relative delays between non scattered and singly forward scattered signals. However, the detection of such a spectral gap at some spectral frequency is very hard or even impossible. The mechanism of colouring here is inverse to the one realized in the FabryPerot interferometer, where monochromatization of radiation results from phase matching of short wave trains. To take account of such matching, a monochromatic wave is formed from a relatively short wave train. In real situations, beside of the mentioned factors, the instrumental factor also influences the observed effect, such as resolving power, spectral sensitivity of detector etc.

We also studied the influence of variations of the dispersion of phase delays in one of the interfering waves on the effectiveness of colouring of the resulting beam. We estimated the result of computer mixing of the colours with different intensities obtained by interference for various phase shifts of the interfering beams. It has been shown that an increase in the dispersion of delays results in a decrease in colouring of the resulting intensity. This result explains the experimental fact that the effectiveness of colouring of a beam that has passed a scattering medium is enhanced in case of a mono-disperse ensemble of light-scattering particles. This clarifies why the occurrences of a blue moon or a blue sun observed in nature are so rare $[12,13]$. 
The effect of narrowing the spectrum of radiation used in the experiment is illustrated in Figure 3(a) and in the interference pattern shown in Figure 3(b). This leads to a decline of the colour gamut at the interferometer output. These peculiarities are illustrated by the fragments in Figure 3(c). One observes a clear shift of the gamut of the resulting colours at

(a)
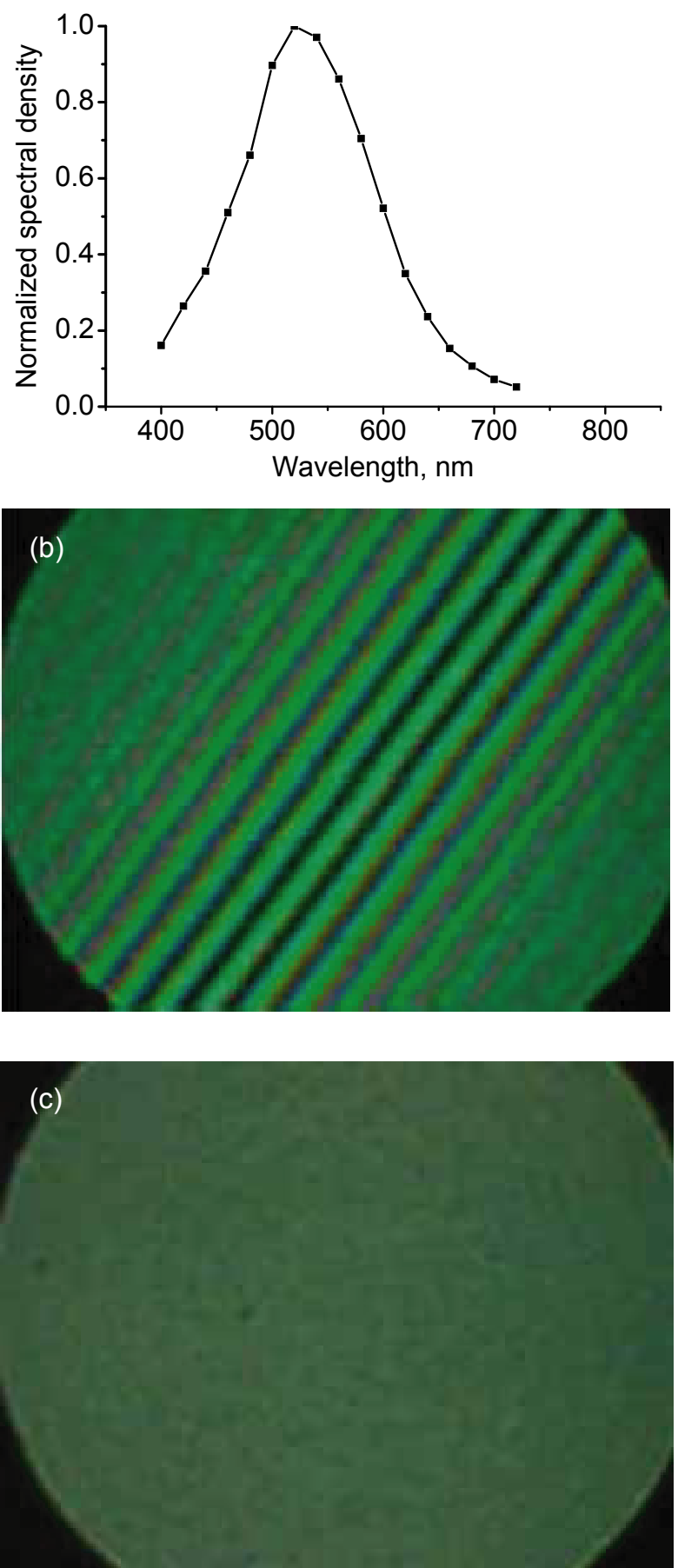

FIG. 3 (a) Spectral content of the used radiation sources. (b) Interferograms obtained with parallel beams in the situation when the angle of convergence of the two waves is different from zero. The presented distributions of fringes qualitatively characterize the spectral content of the interfering beams. The set of colours in the fringes corresponds to the set of colours in a video (Figure. $3(\mathrm{c})$ ) and to the spectrum of the used radiation. (c) The video starts when the optical path difference is comparable with the length of the wave train. The limits of the relative longitudinal scanning of the wave trains are comparable with their lengths. (videosize: 4.7 MB, format: mov, see Fig3c.mov). the interferometer output into the blue-to-green domain, and the colours corresponding to the long-wavelength domain including red are considerably suppressed. These results are in accordance with the spectrum of the used illuminating beam, see Figure 3(a).

(a)
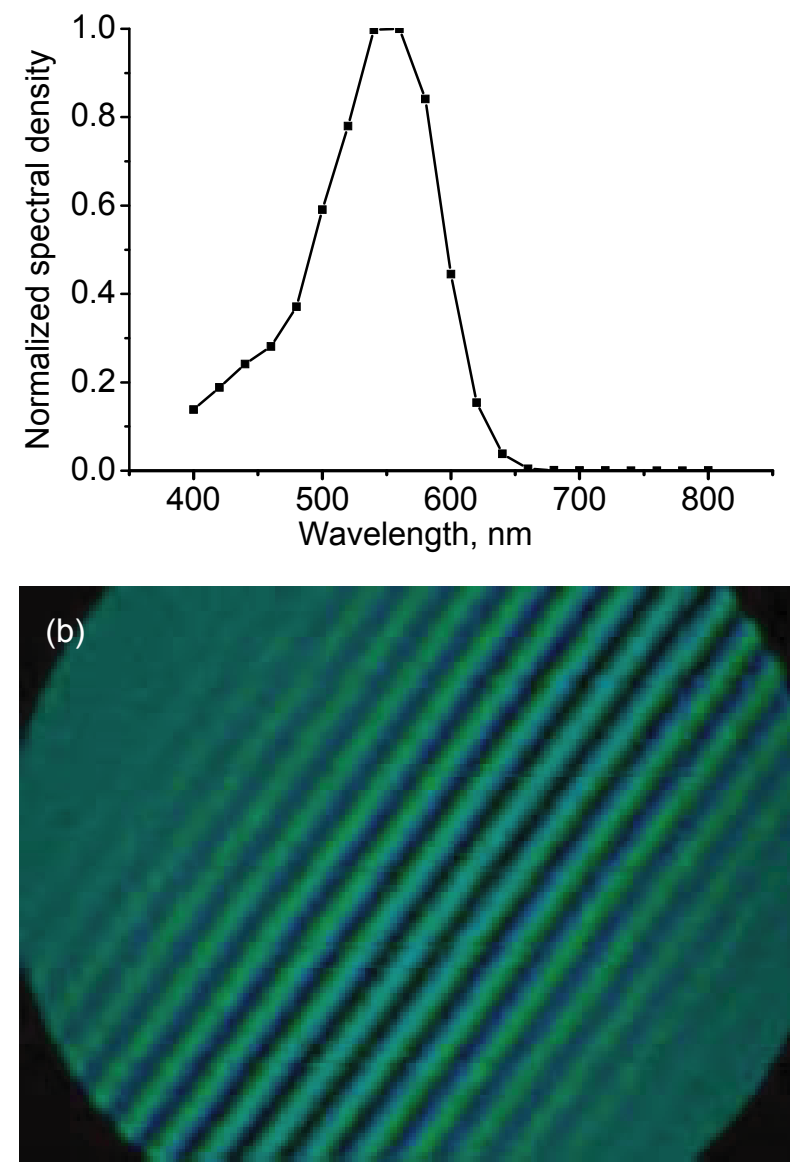

(c)

FIG. 4 (a) Spectral content of the used radiation sources. (b) Interferograms obtained with parallel beams in the situation when the angle of convergence of the two waves is different from zero. The presented distributions of fringes qualitatively characterize the spectral content of the interfering beams. The set of colours in the fringes corresponds to the set of colours in a video (Figure $4(\mathrm{c})$ ) and to the spectrum of the used radiation. (c) The video starts when the optical path difference is comparable with the length of the wave train. The limits of the relative longitudinal scanning of the wave trains are comparable with their lengths. (videosize: $4.8 \mathrm{MB}$, format: mov, see Fig4c.mov). 
The effect of further narrowing of the spectrum is illustrated in Figure. 4(a) and by the interference pattern obtained with no coaxial beams (Figure. 4(b)). In practice, a dichromatic pattern appears at the interferometer output, cf. the fragments of the video (Figure. 4(c)), where one observes only two colours, viz. blue and green. As a matter of fact, the spectrum of the used radiation is characterized by the intensity maximum in the blue-to-green domain, while the long-wavelength spectral domain is considerably suppressed.

In the second step we have studied the effects of colouring of the composite "liquid crystal - polymer matrix". The sample of such composite permits us to study the effects of interference colouring as a function of path difference between the beams passing through polymer and liquid crystal as well as a function of the intensity ratio of such beams. Changing the path difference of the interfering beams was achieved by changing a voltage applied to the cell. Such composites are drops of a liquid crystal (LC) with sizes from 10 to 30 micrometers encapsulated in a polymer matrix (PM).We used a nematic liquid crystalline mixture E7 from the company Merk [18]. As a polymer matrix, we used photopolymer composite NOA65 (production of the Norland Company, USA) that is sensitive to ultraviolet radiation. This polymer matrix was developed for gluing optical elements [19]. NOA65 is widely used together with LC E7 in composites such as LC-PM [20].

The weight parts of the polymer and the liquid crystal are 1:2. The components are carefully mixed at a temperature of $20^{\circ}$. A drop of the mixture is placed between two glass plates covered with conducting ITO films. The thickness of the composite layer is 6 micrometers being controlled by spacers. The cell is assembled with UV-curing glue. Figure 5 shows the area of the studied sample with size 100x75 micrometers in crossed polarisers, without voltage (a) and with applied altering voltage with frequency $f=2000 \mathrm{~Hz}(\mathrm{~b})$. One can see from Figure 5 that the liquid crystalline phase forms disc-like isolated drops whose thickness is controlled by the cell thickness. A histogram of the drop size distribution is presented in Figure 6
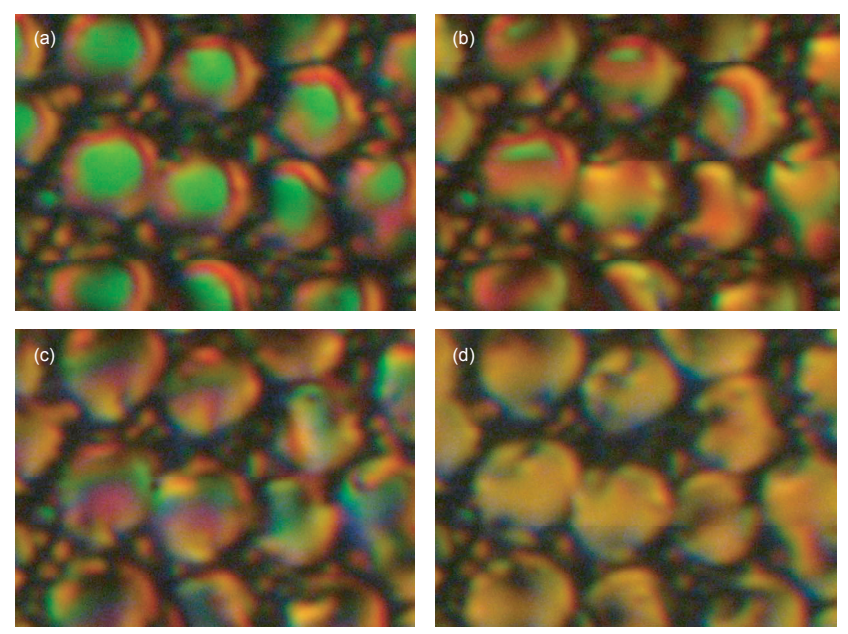

FIG. 5 Microphotographs of the area of the studied sample of sizes $100 \times 75$ micrometers in the crossed polarisers, without (a) applied voltage and with applied altering voltage of frequency $f=2000 \mathrm{~Hz}$ : (b) $2 \mathrm{~V}$, (c) $2.5 \mathrm{~V}$, (d) $3 \mathrm{~V}$.

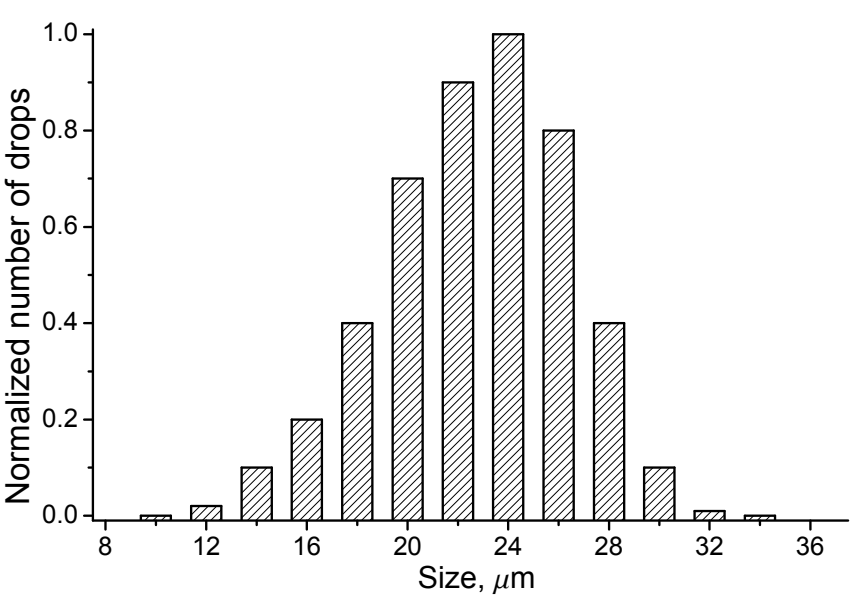

FIG. 6 Histogram of the drop size distribution.

Phase separation results in formation of an optically inhomogeneous medium which strongly scatters the light. The intensity of the scattered light depends on the inhomogeneity of the refractive index inside the liquid crystalline drops, differences in the refractive index between adjacent drops, as well as in the boundaries of the LC and PM phases [21]. The index of refraction of the photopolymer NOA65 $\left(n_{p}=1.524\right)$ is close to the ordinary refractive index of a liquid crystal E7 $\left(n_{0}=1.521\right)$. Birefringence of LC E7 is $\Delta n=0.225$. Generally, molecules in drops of liquid crystal are oriented in such a manner that a radial or a bipolar structure is formed [21, 22]. For bipolar structure, a drop has an optical axis, while for radial structure an optical axis is absent. Bipolar drops without applied voltage are oriented chaotically. In our case, drops are flattened and orienting influence of substrates takes place. Applying an electrical current results in orientation of the optical axes of bipolar drops and molecules of a liquid crystal in radial drops along the direction of the illuminating beam incidence (we consider the case of normal incidence, cf. Figure 5). Increasing the applied voltage results in equalizing the magnitudes of the refraction indices of LC and PM and, hence, in increasing the transmittance of the system. Decreasing the voltage will increase the path difference between the components passing the LC and PM in the forward direction, and thus increase light scattering.

The experimental arrangement is shown in Figure 7. Radiation from the incandescence lamp HL is concentrated at the

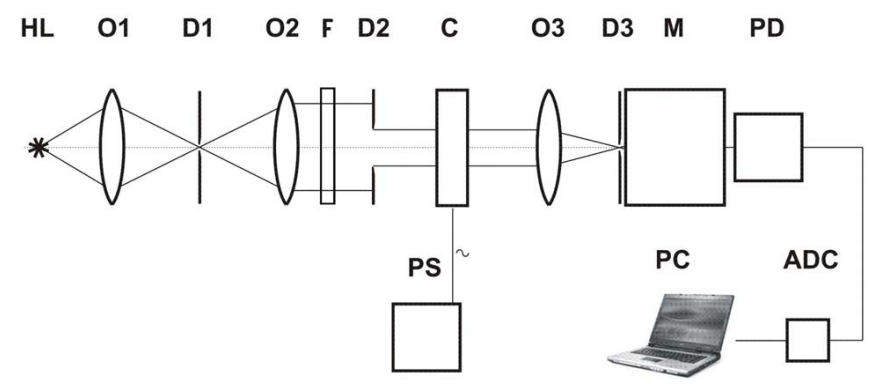

FIC. 7 Experimental arrangement: HL - radiation source, D1, D2, D3 - diaphragms, 01, 02,03 - objectives, $\mathrm{F}$ - spectral filter, C - studied cell, M - monochromator, PD photodetector, ADC - analogue-to-digital converter, PC - computer, PS -power supply. 
diaphragm D1 using a collimator O1. The objective O2 forms a parallel beam. The diameter of the diaphragm $(0.1 \mathrm{~mm})$ and the focal length of the objective $(50 \mathrm{~mm})$ provide a coherence length in the transversal cross-section of the beam of approximately 150 micrometers. Using the filter $\mathrm{F}$, one forms radiation with a desirable spectral content. A parallel beam passes the studied cell $C$ with the composite LC-PM and is focused by $\mathrm{O} 3$ at the diaphragm D3 with diameter $0.1 \mathrm{~mm}$. Such optical system facilitates selection of the regular component of radiation scattered by the composite. The spectrum of the regular component of radiation is measured using a monochromator $\mathrm{M}$. The electrical signal of the photodetector PD through the analogue-to-digital converter (ADC) is transferred to the computer PC. One applies an alternating voltage to the studied cell from the generator PS. It must be emphasized that we use unpolarized optical radiation. The spectrum is shown in Figure 8. Partial beams passing drops of LC and PM pass different paths, and can be considered at far field as plane waves with some phase difference. The far-field conditions are realized using the spatial-frequency filter D1-O2-O3-D3, which separates out the regular component of scattered radiation.

The spectra of radiation passing the cell LC-PM for different applied voltages are shown in Figure 9. The strongest transformation of the spectrum is observed for magnitudes of voltage 2-3.5 V. However, these transformations can not be pronounced due to different intensities of the beams passing the drops LC and PM. Therefore, one can not equalize these intensities by proper choosing the concentration of the components. The reason is that changing of applied voltage results in redistribution between the cell transmittance and light scattering due to changing homogeneity of LC in drops and changing phase difference between the beams. This means that the intensity ratio of the regular beams passed through the drops of LC and PM changes. However, one can estimate the ratio between the intensities of these beams by measuring of the fraction of LC to PM and estimating the influence of the scattered radiation, and computing the resulting spectrum of the part of the regular component on which one of the components predominates. The results of such processing of the data of experimental measurements are represented in Figure 10.

Complete lightening of the cell LC-PM takes place at voltage $8 \mathrm{~V}$ (Figure 10(a)). For a voltage of $3.2 \mathrm{~V}$ one observes

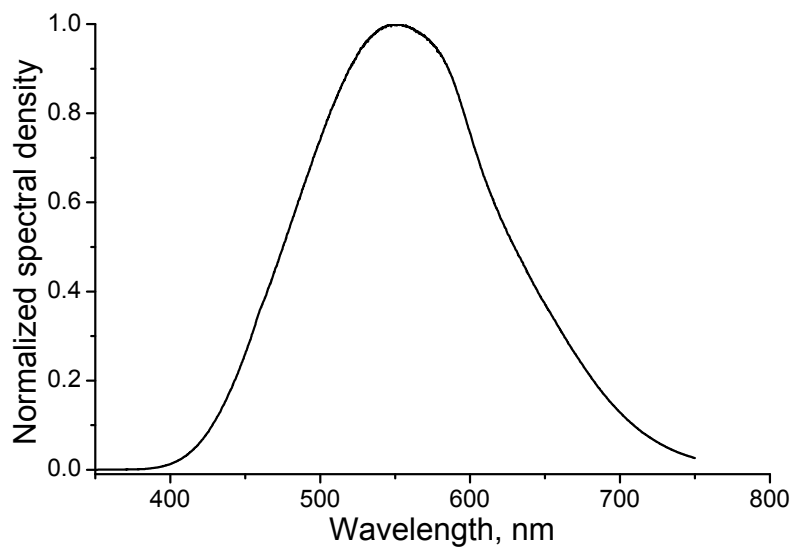

FIG. 8 Spectral characteristics of the illuminating beam.

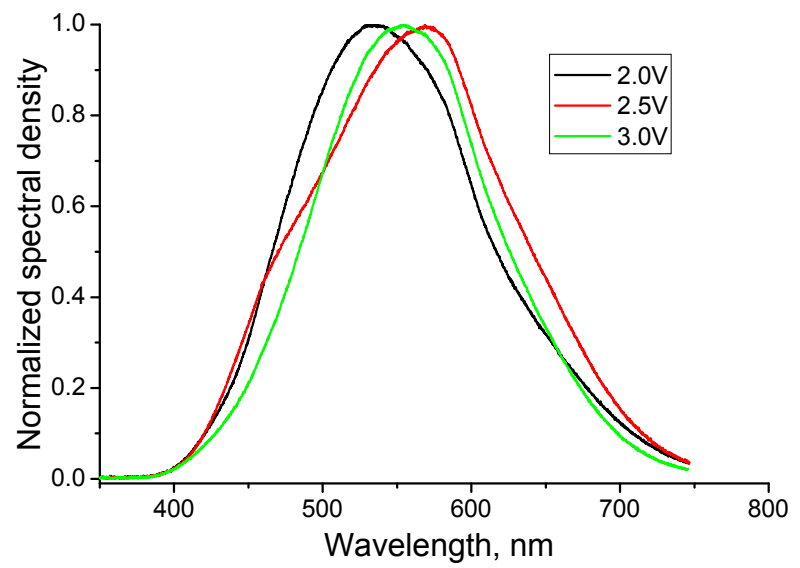

FIG. 9 Spectra of radiation passing the LC-PM cell for applied voltage $2 \mathrm{~V}, 2.5 \mathrm{~V}, 3 \mathrm{~V}$.

a dip in the blue domain of the spectrum (Figure 10(b)). It means that the path difference between two interfering components provides opposite phases of the "blue" components. Microscopic study of the cell LC-PM shows that for the voltage $3.2 \mathrm{~V}$ the corresponding path difference equals $690 \mathrm{~nm}$. From the condition of the opposite phases for the spectral component $3 \lambda_{b} / 2=690 \mathrm{~nm}$, one finds: $\lambda_{b}=460 \mathrm{~nm}$. For a decrease in the applied voltage, the path difference between the interfering beams increases and one observe a shift of the spectral gap from the blue domain to the red one, and re-
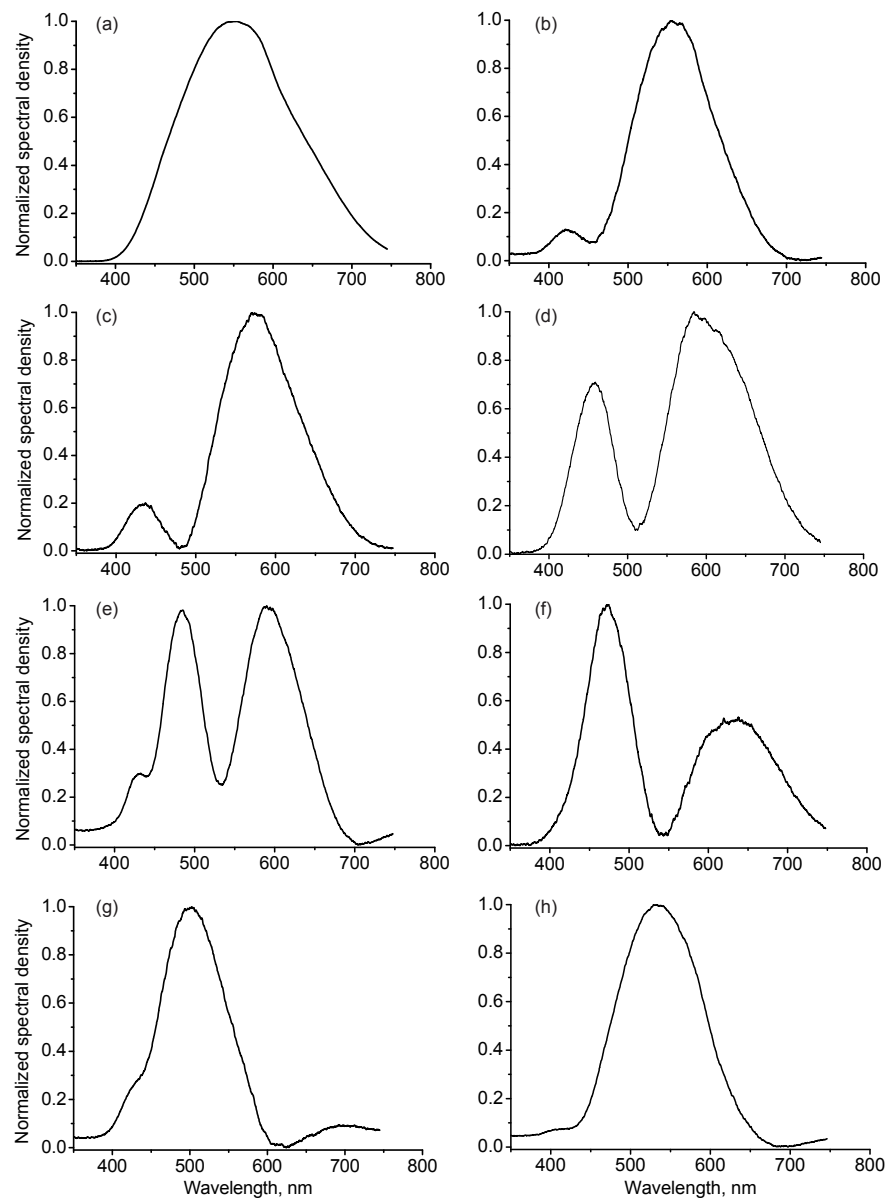

FIG. 10 The processed spectra of radiation passing the LC-PM cell for different applied voltage: $8 \mathrm{~V}(\mathrm{a}), 3.2 \mathrm{~V}(\mathrm{~b}), 3.0 \mathrm{~V}(\mathrm{c}), 2.8 \mathrm{~V}(\mathrm{~d}), 2.4 \mathrm{~V}(\mathrm{e}), 2.2 \mathrm{~V}(\mathrm{f}), 2.0 \mathrm{~V}(\mathrm{~g}), 1.8 \mathrm{~V}(\mathrm{~h})$. 
distribution of the heights of spectral maxima (Figure 10(c)(h)). In the last figure, Figure 10(h), one observes simultaneous minima for the red and the blue spectral components. In this case, the condition of opposite phases for the red spectral component is: $3 \lambda_{r} / 2=1020 \mathrm{~nm}$, and for the blue component is: $5 \lambda_{b} / 2=1020 \mathrm{~nm}$. Thus, one finds the wavelengths of the corresponding spectral components: $\lambda_{r}=680 \mathrm{~nm}$, and $\lambda_{b}=408 \mathrm{~nm}$. In other words, one can see considerable narrowing down of the half-width of the spectrum of the resulting radiation in comparison with the spectrum of the illuminating beam. The origin of this narrowing is simply interference.

\section{CONCLUSIONS}

We have illustrated the interference origin of the mechanisms of colouring of radiation passing through a light-scattering medium in the forward direction. It has been shown that the colouring of the output beam from a light-scattering medium depends on the magnitudes of the phase delays of singly scattered forward partial signals. The amplitude ratio of nonscattered and singly forward scattered interfering components also significantly affects the colour intensity. It has been established that the spectral content of the illuminating beam strongly affects the colour of the resulting radiation. So, we have for the first time shown the possibility for obtaining a bright green colouring of the beam at the output of a lightscattering medium, which is caused by simultaneous destructive interference of the components in the near- and the farwavelength domains of the visible spectrum of the used radiation. All the mentioned effects show once more that the effect of colouring of radiation passing through a light-scattering medium, irrespectively from its nature (disperse media, suspensions of particles in air, rough surfaces, hoar-frost at a window), is the result of interference, i.e. singular-optical subtraction of the spectral components from the spectrum of the illuminated beam.

\section{ACKNOWLEDGEMENTS}

S.G. Hanson acknowledges financial support from the Danish Council for Technology and Innovation under the Innovation Consortium CINO (Centre for Industrial Nano Optics).

\section{References}

[1] G. Gbur, T.D. Visser, and E. Wolf, "Anomalous behavior of spectra near phase singularities of focused waves" Phys. Rev. Let. 88, 013901 (2002).

[2] G. Popescu, and A. Dogariu, "Spectral anomalies in wave-front dislocations" Phys. Rev. Let. 88, 183902 (2002).

[3] J. Leach, and M.J. Padgett, "Observation of chromatic effects near a white light vortex" New J. Phys. 5, 1-7 (2003).
[4] M.M. Brundavanam, N.K. Viswanathan, and N.R. Desai, "Spectral anomalies due to temporal correlation in a white-light interferometer" Opt. Lett. 32, 2279-2281 (2007)

[5] A. Ponomarenko, and E. Wolf,"Spectral anomalies in a Fraunhofer diffraction pattern" Opt. Lett. 27, 1211-1213 (2002).

[6] 0. V. Angelsky, S.G. Hanson, A.P. Maksimyak, and P.P. Maksimyak, "On the feasibility of determining the amplitude zeroes in polychromatic fields" Opt. Express 13, 4396-4405 (2005)

[7] 0.V. Angelsky, S.G. Hanson, A.P. Maksimyak, and P.P. Maksimyak, "Interference diagnostics of white light vortices" Opt. Express 13, 8179-8183 (2005).

[8] P.V. Polyanskii, "Optical correlation diagnostics of phase singularities in polychromatic fields" in Optical Correlation Applications and Techniques, 0. Angelsky, ed., (SPIE Press A168, Bellingham, 2007).

[9] V. Shvedov, W. Krolokowski, A. Volyar, D.N. Desyatnikov, and Y.S. Kivshar, "Focusing and correlation properties of white-light optical vorices" Opt. Express 13, 7393-7398 (2005).

[10] V.K. Polyanskii, 0.V. Angelsky, and P.V. Polyanskii, "Scatteringinduced spectral changes as a singular-optical effect" Opt. Appl. 32, 843-848 (2002).

[11] 0.V. Angelsky, P.V. Polyanskii, and S.G. Hanson, "Singular-optical coloring of regularly scattered white light" Opt. Express. 14, 75797586 (2006).

[12] C.F. Bohren, and D.R. Huffman, Absorption and scattering of light by small particles (Wiley, New York, 1983).

[13] M. Minnaert, The Nature of Light and Colour in the Open Air (Dover, New York 1954).

[14] L.V. Strinadko, V.K. Polyanskii, and M.T. Strinadko, "On the structure of the field scattered by a phase transparency" opt. Spectrosc. 49, 952-957 (1980).

[15] 0.V. Angelsky, A.P. Maksimyak, P.P. Maksimyak, and S.G. Hanson, "Optical correlation diagnostics of rough surfaces with large surface inhomogeneities" Opt. Express 14, 7299-7311 (2006).

[16] M. Berry, "Coloured phase singularities" New J. Phys. 4, 66.1-66.14 (2002).

[17] M. Berry, "Exploring the colour of dark light" New J. Phys. 4, 74.174.14 (2002).

[18] P. Mormile, L. Petti, M. Abbate, P. Musto, G. Ragosta, and P. Villano, "Temperature switch and thermally induced optical bistability in a PDLC" Opt. Commun. 147, 269-273 (1998).

[19] Technical Data Sheet for N0A65 Optical Adhesive, Norland Products Inc., North Brunswick, NJ.

[20] J.-J. Wu, and C.-M. Wang, "Electro-optical properties of aligned polymer dispersed liquid crystal films" Phys. Lett. A 232, 149-154, (1997).

[21] L. Bouteiller, and P. Le Barny, "Polymer-dispersed liquid crystals: Preparation, operation and application" Liq. Cryst. 21, 157-174 (1996).

[22] D. Higgins, "Probing the Mesoscopic Chemical and Physical Properties of Polymer-Dispersed Liquid Crystals" Adv. Mater. 12, 251-264 (2000). 